\title{
A Symbol Rate Blind Estimation Algorithm of Baseband Signal
}

\author{
Yuexuan Liu, Jiangang Wang, Xiaohui Li, Longxing Tang and Yuehuai Ma
}

Training Department, PLA Communication Training Base of GSD, Zhangjiakou, Hebei, China (yuexuanliu@ 163.com)

\begin{abstract}
Accurate estimation of symbol rate is an important part of the electronic information countermeasures. In this article, the cyclic spectrum density expression of band-limited baseband signal is derived. And analysed it's characteristics. A new blind estimation algorithm of symbol rate based on cyclic spectrum is presented. This algorithm don't need the information of modulation type. It is insensitive of carrier frequency offset, and have good performance in low SNR. The effectiveness of this algorithm is proved by computer simulations.
\end{abstract}

Keywords — electronic countermeasures, symbol rate, cyclic spectrum, blind estimation

\section{一种基带信号符号速率盲估计算法}

刘跃宣 王建刚 李晓辉 汤隆兴 马月槐

总参通信训练基地训练部，张家口，河北，中国

摘 要 符号速率的准确估计是电子信息对抗的重要环节。推导出带限基带信号循环谱表达式并分析了其特点, 提出一种基于循 环谱分析的符号速率盲估计方法。该方法不需要知道信号调制类型, 对载波频偏不敏感, 低信噪比下仍有较好的性能。仿真验证了其 有效性。

关键词 电子对抗, 符号速率, 循环谱, 盲估计

\section{1. 引言}

对于电子对抗、软件无线电等非协作通信, 对其各种 通信参数的估计是首要和必须的, 而且需要进行盲估计。 符号速率是通信中的重要参数。符号速率的准确估计, 是 实施解码、侦听、通信干扰等工作的前提和基础, 是电子 信息对抗的一个重要环节, 也是软件无线电接收机的一个 重要的组成部分。文献[1]利用信号复包络频谱分析的方法 进行符号速率估计, 能很好的适用于 PSK 和 QAM 信号。 文献[2][3]分别用余弦窗变换的方法和小波变换的方法对 MPSK 和 QAM 信号进行符号速率估计。但这些算法抗噪 声性能较差, 在低信噪比下性能不好。

文献[4]中提出了数字调制方式因为符号周期而表现 出循环平稳特性, 可以根据循环平稳分析来估计符号率。 因为在循环频率不等于零处高斯白噪声的循环谱为零, 故 这种方法有很强的抗噪声性能。文献[5][6][7][8]利用循环谱 理论对数字信号的符号速率估计算法做了研究, 但都是针 对射频信号的分析, 这就需要系统有很高的抽样速率, 进 而导致运算量大大增加。

本文推导出了经过正交下变频的带限基带信号循环
谱, 并分析了其特点。进而提出了一种针对带限基带信号 循环谱分析的符号速率估计算法, 利用对信号循环谱的二 维搜索, 在未知调制方式的条件下进行码元速率的估计, 适用的调制方式为 MASK、MPSK、QAM。该算法有很强 的抗噪声性能, 且由于该算法处理对象为基带信号, 系统 抽样速率可以大大降低, 其运算量也就可以大大减少, 对 工程应用和实时处理有重要意义。

\section{2. 循环谱基本理论}

传统的信号模型都是以平稳随机过程为基础的, 但实 际的数字通信信号更适合用循环平稳随机过程模型来描 述。与传统的谱分析法相比, 循环谱可以提供更丰富的信 息, 且抗干扰能力强。

若二阶连续零均值非平稳随机信号 $\{x(t), t \in(-\infty, \infty)\}$ 的 自相关函数 $R_{x}(t, T)$ 呈现以 $T$ 为周期的周期性, 则称 $x(t)$ 为循 环平稳信号。其循环自相关函数表示为:

$$
R_{x}^{\alpha}(\tau)=\frac{1}{T} \int_{-T / 2}^{T / 2} R_{x}(t, \tau) e^{-j 2 \pi \alpha t} d t
$$

式中 $\alpha$ 为循环频率。(1)式的傅立叶变换称为循环谱密 
度函数, 也叫谱相关函数, 简称循环谱, 表示为:

$$
S_{x}^{\alpha}(f)=\int_{-\infty}^{\infty} R_{x}^{\alpha}(\tau) e^{-j 2 \pi f \tau} d \tau
$$

当信号 $x(t)$ 通过一个单位冲击响应为 $h(t)$ 的线性时不变 系统时, 输出信号 $z(t)$ 为:

$$
z(t)=h(t) \otimes x(t)=\int_{-\infty}^{\infty} h(u) x(t-u) d u
$$

其中 “ $\otimes ”$ 表示卷积, 若 $h(t)$ 的傅立叶变换为 $H(f)$, 信号 $x(t)$ 的谱相关密度函数为 $S_{x}^{\alpha}(f)$, 则 $z(t)$ 的谱相关密度 函数有 ${ }^{[9]}$ :

$$
S_{z}^{\alpha}(f)=H\left(f+\frac{\alpha}{2}\right) H^{*}\left(f-\frac{\alpha}{2}\right) S_{x}^{\alpha}(f)
$$

循环谱的估计一般都是通过循环周期图平滑处理后得 到的。具体来说有离散频率平滑方法和离散时间平滑方法。 本文采用离散频率平滑方法, 由下式给出:

$$
\begin{aligned}
& \tilde{S}_{x \Delta t}^{\alpha}(t, f)_{\Delta f}= \\
& \frac{1}{M} \sum_{v=-(M-1) / 2}^{(M-1) / 2}\left[\frac{1}{\Delta t} \tilde{X}_{\Delta t}\left(t, f+\frac{\alpha}{2}+v F_{s}\right) X_{\Delta t}^{*}\left(t, f-\frac{\alpha}{2}+v F_{s}\right)\right]
\end{aligned}
$$

式中 $\Delta f=M F_{s}$ （其中 $F_{s}=1 /\left(N T_{s}\right)$ ) 是谱平滑间隔, $\Delta t=(N-1) T_{s}$ 是数据总长度, 且:

$$
\tilde{X}_{\Delta t}(t, f)=\sum_{k=0}^{N-1} W_{\Delta t}\left(k T_{s}\right) x\left(t-k T_{s}\right) e^{-j 2 \pi\left(t-k T_{s}\right)}
$$

其中 $W_{\Delta t}\left(k T_{s}\right)$ 是数据窗函数, 且其可靠性条件为: $\Delta t \Delta f \approx M>>1$ 。

\section{3. 信号模型及其循环谱}

对射频信号载波频率和带宽的粗略估计是比较容易 的, 进行正交下变频也是比较容易实现的。所以, 本文把 经过正交下变频的基带信号作为处理对象。此时的信号存 在载波频偏和相位差, 码元速率未知且位定时误差未知, 并存在加性高斯白噪声。信号模型复数形式(包括 MASK、 MPSK、QAM 信号)可表示为:

$$
r(t)=\left(\sum_{n=-\infty}^{\infty} b_{n} g_{T}\left(t-n T_{b}-\varphi_{0}\right)\right) \cdot e^{j\left(2 \pi \Delta t t+\theta_{0}\right)}+n(t)
$$

其中 $g_{T}(t)$ 为频域具有平方根升余弦滚降特性的成型滤 波器的单位冲击响应, $\left\{b_{n}\right\}$ 为复数据序列, 且 $E\left(b_{n}\right)=0, E\left(b_{n} b_{m}^{*}\right)=B^{2} \delta_{n, m}$ (我们称具有这种性质的序列 为白振幅序列 ); $\varphi_{0}$ 为定时误差; $T_{b}$ 为符号间隔; $\Delta f$ 为残 留载波频偏; $\theta_{0}$ 为载波相位差; $n(t)$ 是均值为 0 、方差为 $\sigma^{2}$ 的复高斯白噪声。

对于白振幅数据序列 $\left\{b_{n}\right\}$, 文献[5]指出其循环谱为:

$$
S_{b}^{\alpha}(f)=\left\{\begin{array}{ccc}
R_{b}(0) & \alpha=k & T_{b} \\
0 & \alpha \neq k / T_{b}
\end{array}\right.
$$

为便于比较, 我们先求成型脉冲为矩形脉冲 $q(t)$ 的 PAM(Pulse Amplitude Modulation)信号的循环谱。令:

$$
A(t)=\sum_{n=-\infty}^{\infty} b_{n} q\left(t-n T_{b}\right)
$$

PAM 信号相当于数据序列 $\left\{b_{n}\right\}$ 通过单位冲击响应为矩 形脉冲 $q(t)$ 的成型滤波器的输出, 据(4)式得其循环谱为:

$$
S_{A}^{\alpha}(f)=Q\left(f+\frac{\alpha}{2}\right) Q^{*}\left(f-\frac{\alpha}{2}\right) S_{b}^{\alpha}(f) \quad \alpha=\frac{k}{T_{b}}
$$

式中 $k$ 为整数, $Q(f)$ 为矩形脉冲 $q(t)$ 的傅立叶变换, $Q(f)=\sin \left(f T_{b}\right) /(\pi f)$, “*” 表示复数共轭。

当信号为带限信号时, 考虑成型脉冲为 $g_{T}(t)$ 的 PAM 信 号:

$$
B_{0}(t)=\sum_{n=-\infty}^{\infty} b_{n} g_{T}\left(t-n T_{b}\right)
$$

据(4)式得其循环谱为:

$$
\begin{aligned}
& S_{B 0}^{\alpha}(f)=G_{T}\left(f+\frac{\alpha}{2}\right) G_{T}^{*}\left(f-\frac{\alpha}{2}\right) S_{b}^{\alpha}(f) \\
& \text { (其中 } \alpha=\frac{k}{T_{b}}, k=0, \pm 1 \text { ) }
\end{aligned}
$$

其中 $G_{T}(f)$ 为 $g_{T}(t)$ 的傅里叶变换, 为:

$$
G_{T}(f)= \begin{cases}\sqrt{T_{b}} & |f| \leq \frac{1-\alpha}{2 T_{b}} \\ \sqrt{T_{b}} \cos \left[\frac{\pi}{4 \alpha}\left(\left|2 f T_{b}\right|-1+\alpha\right)\right] & \frac{1-\alpha}{2 T_{b}} \leq|f| \leq \frac{1+\alpha}{2 T_{b}} \\ 0 & \text { else }\end{cases}
$$

对 $B_{0}(t)$ 延时 $\varphi_{0}$ 的信号 $B(t)$ 为:

$$
B(t)=B_{0}\left(t-\varphi_{0}\right)=\sum_{n=-\infty}^{\infty} b_{n} g_{T}\left(t-n T_{b}-\varphi_{0}\right)
$$

若 $B_{0}(t)$ 的循环自相关函数为 $R_{B 0}^{\alpha}(\tau)$, 则 $B(t)$ 的循环 自相关函数为 ${ }^{[6]} R_{B}^{\alpha}(\tau)=R_{B 0}^{\alpha}(\tau) e^{-j 2 \pi \alpha \varphi_{0}}$, 则(7)式所示信 号 $r(t)$ 的循环自相关函数为 (无噪声时) $R_{r}^{\alpha}(\tau)=R_{B}^{\alpha}(\tau) e^{j 2 \pi \Delta f \tau}$ 。据傅里叶变换的性质, 信号 $r(t)$ 的循环谱 $S_{r}^{\alpha}(f)$ 为:

$$
\begin{aligned}
& S_{r}^{\alpha}(f)=S_{B}^{\alpha}(f-\Delta f)=S_{B 0}^{\alpha}(f-\Delta f) e^{-j 2 \pi \alpha \varphi_{0}} \\
& \text { (其中 } \left.\alpha=\frac{k}{T_{b}}, k=0, \pm 1\right)
\end{aligned}
$$

对于 $(12)$ 式和(15)式, 因 $G_{T}(f)$ 具有平方根升余弦滚 
降特性, 其带宽不大于 $1 / T_{b}$, 故只有当 $k=0, \pm 1$ 时才有值。

可以看出对于调制方式为 MASK、MPSK、QAM 的基 带信号, 其循环谱形状是相同的, 只不过幅值有所不同, 故本文的第 6 部分的性能分析仿真均以 $B P S K$ 为例。

\section{4. 信号的循环谱特性分析}

\section{1 矩形 PAM 信号的循环谱}

(9)式所示信号 $A(t)$ 的循环谱 $S_{A}^{\alpha}(f)$ 如图 1 所示。由于 矩形脉冲的频谱旁瓣较大, 所以当 $|k|$ 增大时 $S_{A}^{\alpha}(f)$ 衰减较 慢。在循环域内, 当 $\alpha=k / T_{b}, k=0, \pm 1, \pm 2, \ldots$ 时, $S_{A}^{\alpha}(f)$ 有非零值。循环谱的最大值出现在 $\alpha=0, f=0$ 处。 $\alpha \neq 0$ 时的 最值出现在 $f=0, \alpha= \pm 1 / T_{b}$ 处。

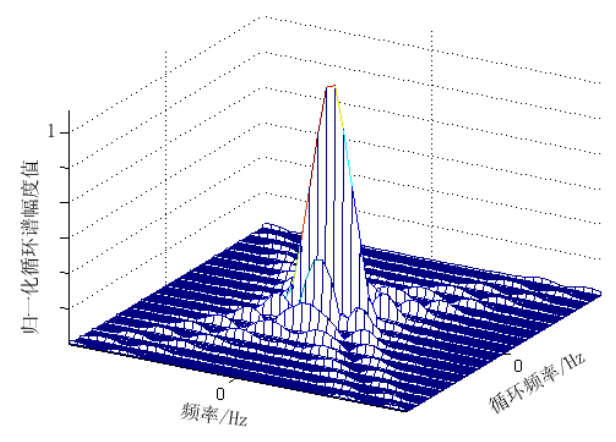

图 $1 A(t)$ 的循环谱

\section{2 带限 PAM 信号的循环谱}

本文考虑成型滤波器具有频域平方根升余弦滚降特 性, 其单位冲击响应 $g_{T}(t)$ 。图 2 和图 3 为滚降系数 $\alpha=1$ 和 $\alpha=0.5$ 时(11)式所示信号 $B_{0}(t)$ 的循环谱 $S_{B 0}^{\alpha}(f)$ 。由于 $g_{T}(t)$ 带宽受限, 最大带宽为 $1 / T_{b}$ (当滚降系数为 1 时), 所以, 在循环域, 仅当 $\alpha=k / T_{b}, k=0, \pm 1$ 时, $S_{B 0}^{\alpha}(f)$ 有非零值。 而且, 当 $k= \pm 1$ 时 $S_{B 0}^{\alpha}(f)$ 的值随着 $g_{T}(t)$ 滚降系数的减小 而减小。

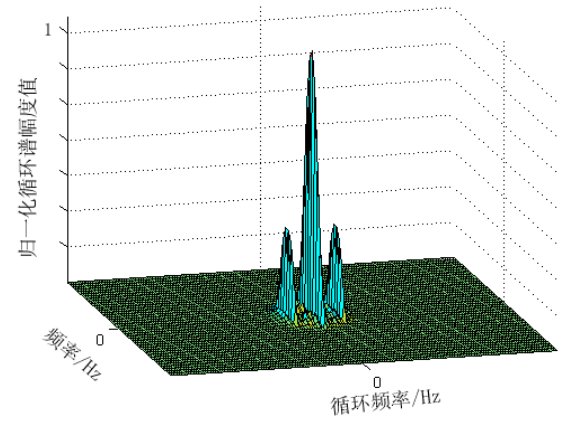

图 $2 A(t)$ 的循环谱

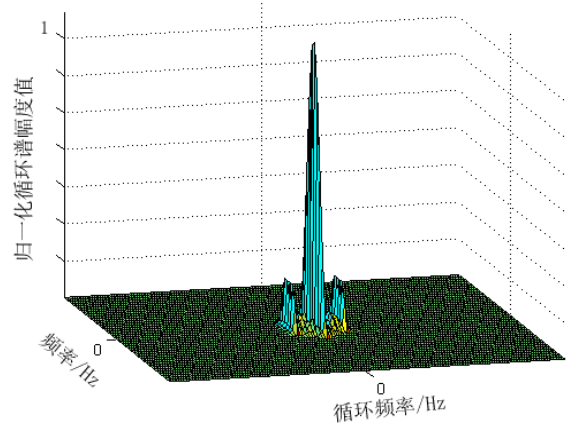

图 $3 B_{0}(t)$ 的循环谱

4.3 有频偏和相偏信号的循环谱 (无噪声时)

无噪声时 (7)式所示信号 $r(t)$ 的谱相关函数 $S_{r}^{\alpha}(f)$ 为 $S_{B 0}^{\alpha}(f)$ 频移 $(-\Delta f)$ 再乘以系数 $e^{-j 2 \pi \alpha Q}$, 所以 $S_{r}^{\alpha}(f)$ 与 $S_{B 0}^{\alpha}(f)$ 相比形状不变, 只是位置和幅值有所变化。循环域 的特性与 $S_{B 0}^{\alpha}(f)$ 相同。图 4 为无噪声时 $r(t)$ 的循环谱。可 见循环谱的最大值出现在 $\alpha=0, f=\Delta f$ 处。 $\alpha \neq 0$ 时的最值出 现在 $f=\Delta f, \alpha= \pm 1 / T_{b}$ 处, 且 $\alpha$ 为其他值时循环谱为零。

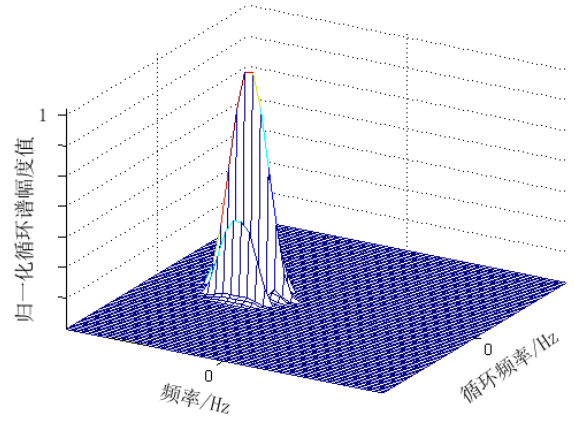

图 $4 r(t)$ 的循环谱

\section{4 信号加噪声的循环谱}

因为高斯白噪声 $n(t)$ 为均值为零的平稳随机过程, 不具 有非零的循环频率[5], 因此噪声只对循环谱的循环频率 $\alpha$ 为零处有影响, $\alpha \neq 0$ 时噪声的循环谱为零。图 5 和图 6 为 高斯白噪声 $n(t)$ 的循环谱和混有噪声的信号 $r(t)$ 的循环谱。

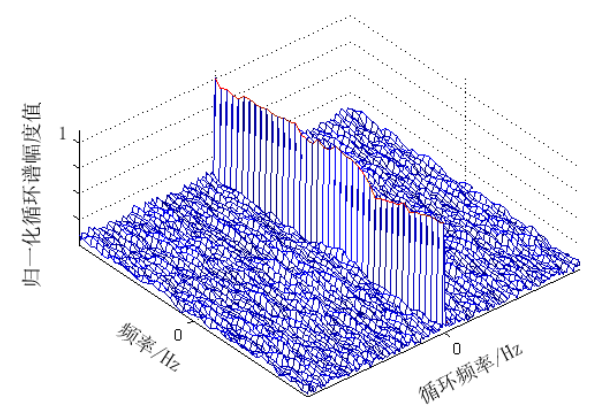

图 5 高斯白噪声 $n(t)$ 的循环谱 


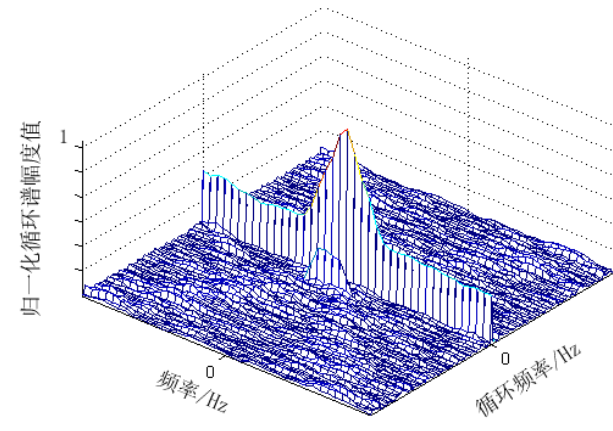

图 6 混有噪声的信号 $r(t)$ 的循环谱

\section{5. 基于循环谱的符号速率估计算法}

算法步骤如下:

1、由信号 $r(t)$ 采样得到序列 $r(n)$, 取长度为 $N$ 点的序 列 $r_{N}(n)$ 。

2、计算循环频率 $\alpha \neq 0$ 时的谱相关函数 $S_{r}(m, 0)$, 其 中 $m$ 对应 $f$ 域, 0 对应 $\alpha$ 域。

3、搜索 $\alpha=0$ 时, $S_{r}(m, 0)$ 在 $f$ 域的最大值, 得到最大 值对应的频率 $f=f_{0}$ 。 $f_{0}$ 为频偏的估计值。

4、计算 $f=f_{0}$ 时的谱相关函数 $S_{r}\left(f_{0}, n\right)$ 。其中 $f_{0}$ 对应 $f$ 域, $n$ 对应 $\alpha$ 域。

5、搜索 $f=f_{0}$ 时, $S_{r}\left(f_{0}, n\right)$ 在 $\alpha>0$ 范围内最大值, 得 到最大值对应的循环频率 $\alpha=f_{b}$, 此即为符号速率的估计 值。

\section{6. 算法分析及性能仿真}

算法第 3 步中, 当 $\alpha=0$ 时的循环谱 $S_{r}(m, 0)$ 为噪声谱 与信号谱的叠加, 噪声对最大值搜索影响不大。所以, 我 们主要研究后续步骤的性能。

为了衡量上述第 5 步中 $\alpha=1 / T_{b}$ 时所对应的峰值的明 显性, 我们定义参数 PPD $(\mathrm{dB})$ (Peak Power Difference):

$$
\operatorname{PPD}(d B)=10 \log _{10}\left(A_{1} / A_{2}\right)
$$

其中 $A_{l}$ 表示在 $f=f_{0}$ 时的 $\alpha$ 域 $(\alpha>0)$ 内, $\alpha=1 / T_{b}$ 所对应 的峰值; $A_{2}$ 表示除了 $A_{1}$ 外的峰值。下面我们分析算法性能。

\section{1 抗噪声性能}

由于循环频率不为零时, 宽带高斯白噪声的影响理论 上为零, 该算法有较好的抗噪声性能。具体计算时有误差, 但随着取的数据长度的增加, 噪声的影响会趋于零。图 7 为 $\operatorname{SNR}\left(E_{b} / n_{0}\right)$ 为 $-2 \mathrm{~dB} \sim 4 \mathrm{~dB}$ 时, 信号循环谱的 PPD 参数的平 均值, 仿真条件为取 500 个符号, 抽样率为 8 倍符号速率, 成形滤波器滚降系数为 0.7 , 每种信噪比下重复运算 25 次 取平均。可以看出, 信噪比为 $-1 \mathrm{~dB}$ 时, 信号的 PPD 参数就
达到了 $0 \mathrm{~dB}$ 左右。而用文献[2]中的方法, 达到同等性能要 求信噪比为 $6 \mathrm{~dB}$ 左右。可见此方法提高了 $7 \mathrm{~dB}$ 左右。

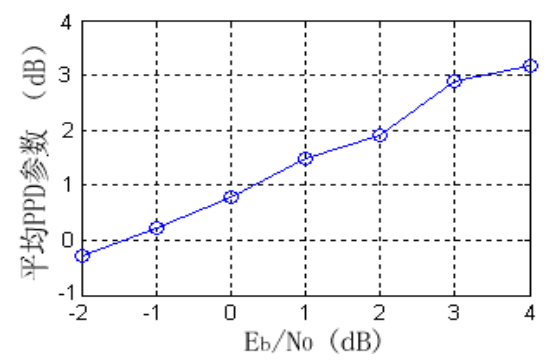

图 7 信号平均 PPD 参数

\section{2 估计精度}

此方法的频率分辨率为 $2\left(f_{s} / N\right)$, 其中 $f_{s}$ 为抽样速率, $N$ 为观测数据总长度。估计误差不大于频率分辨率。若取数 据长度为 1000 个符号, 则估计精度在 $10^{-3}$ 数量级。要想提 高精度, 需以增加样本数据长度为代价。

\section{参考文献(References)}

[1] B.S. Koh, H.S. Lee, "Detection of Symbol rate of Unknown Digital Communication Signals," Electronic Letters, Vol.29, No.3, pp.278-279, 1993.

[2] Zheng Wen-xiu, Yang Shao-quan, Zhao Guo-qing etc. "Symbol Rate Estimation Based on Cosine Window Transform for MPSK and QAM Signals," Electronic Information Warfare Technology, vol.21, no.5, pp.7-10, 2006.

[3] Luo Ming, Yang Shao-quan, Zheng Wen-xiu etc. "On Symbol Rate Estimation for Signal Reconnaissance," Electronic Warfare Technology, vol.20, no.5, pp.3-6, 2005.

[4] Gardner W.A, "Signal interception: a unifying theoretical framework for feature detection". Communications, IEEE Transactions on, vol.36, no.8, pp.897-906, 1988.

[5] Zhang Zi-bing, Yang Kun-ming, Yu Tian-sheng, “The technology research of detection and parameters estimation of the non-cooperative communication signals based on the cyclostationary theory: part 1 fundamental and algorithm," Journal of chongqing communication institute, vol.23, no.4, pp.63-68, 2004.

[6] Tang Su-hu, Dai Xu-ch, Xu Pei-xia, etc. "Cyclostationarity-based Symbol Rate Estimation," Acta Armamentarii, vol.26, no.2, pp.108-112, 2005.

[7] Xie Ran, Zhang Xiao-yi, Tian Peng-wu, "Symbol rate estimation method of MPSK signals based on cyclic spectrum," Application Research of Computers, vol.28, no.6, pp.2294- 2296, 2011.

[8] Zheng Peng, Liu Feng, Zhang Xin, etc. "An Improved Algorithm for Detection and Chip-Rate Estimation for MPSK Signal Based on Cyclic Spectrum," Electronics Optics \& Control, vol.19, no.8, pp.41-44, 2012. 